\title{
Feedforward Control of Quadratic Converters
}

\author{
FELIX A. HIMMELSTOSS, HELMUT L. VOTZI \\ Electronics Department \\ University of Applied Sciences Technikum Wien \\ Hoechstaedtplatz 6 \\ AUSTRIA
}

\begin{abstract}
DC/DC converters, which have a quadratic term of the duty cycle, are useful, when wide voltage transformation ratios have to be obtained. Taking a d-square step-down converter as an example, the function of the converter, the generation of the large and small signal models is explained. From the voltage transformation ratio, a control law is derived and the behavior of the feed-forward converter is shown by simulations. Results of two other quadratic converters are also shown.
\end{abstract}

Key-Words: - DC/DC converters, quadratic, feedforward control, modelling, step-down, control law

Received: January 3, 2020. Revised: June 14, 2021. Accepted: June 22, 2021. Published: July 2, 2021.

\section{Introduction}

DC/DC converters, which have a quadratic term of the duty cycle, are useful, when wide voltage transformation ratios have to be obtained. The classical paper concerning this topic is [1]. One interesting fact is that these converters need only one active switch and the other three necessary switches are realized by diodes. It should be mentioned that one could substitute these diodes by active switches for low voltages to reduce losses, but this increases the cost because of the necessary drivers and the additional control expense.

Basic information about DC/DC converters can be found in the textbooks e.g. [2-4]. Other topologies can be found in the topology studies [5-7]. Quadratic converters with coupled coils are treated especially in [8-11]. In this paper we explain the function, the modelling and the feedforward control of a quadratic converter for an example. Simulation results are shown also for two other converters to show the effectivity of the feedforward concept.

\section{Function of the D-Square Step- Down Converter}

In this chapter we explain the operation of the dsquare step-down converter according to the circuit diagram shown in Fig. 1. The converter is shown with a resistive load. This converter is also useful as a driver for a permanent magnet DC machine, as battery charger or as a converter for supplying LEDs. Two modes exist in the continuous inductor current mode (CICM). In mode M1 the active switch $\mathrm{S}$ and the diode $\mathrm{D} 1$ are conducting and mode M2 starts, when the active switch S1 is turned off.
Now also $\mathrm{D}_{1}$ turns off and the current through $\mathrm{L}_{1}$ commutates into $\mathrm{D}_{2}$ and the current through $\mathrm{L}_{2}$ commutates into $\mathrm{D}_{3}$.

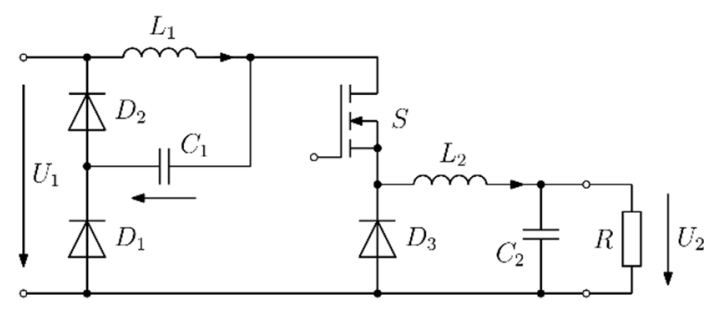

The best way to get to know a converter is to take a sheet of chequered paper, a pencil and an eraser and draw the signals of the converter. Idealized devices, steady state, CICM and appropriate large capacitors, so that the voltage across them stays constant within one switching period, are assumed. The best way to start with are the voltages across the inductors. With a duty cycle of one third, we get the voltages across the inductors according to Fig. 2.

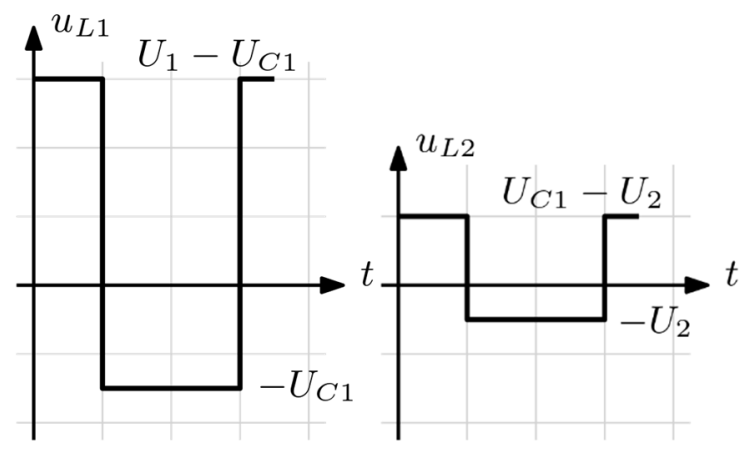

From the voltage-time balance across the inductors (the voltage across the inductors must be zero in the steady state mode) one gets 


$$
\begin{aligned}
& U_{C 1}=d U_{1} \\
& U_{2}=d U_{C 1} .
\end{aligned}
$$

This leads to the voltage transformation ratio

$$
\frac{U_{2}}{U_{1}}=d^{2}
$$

Now one can start to draw the voltages across the semiconductors. With Kirchhoff's voltage law one gets the signals according to Fig. 3. Now one knows the necessary voltages for the active and the passive switches.
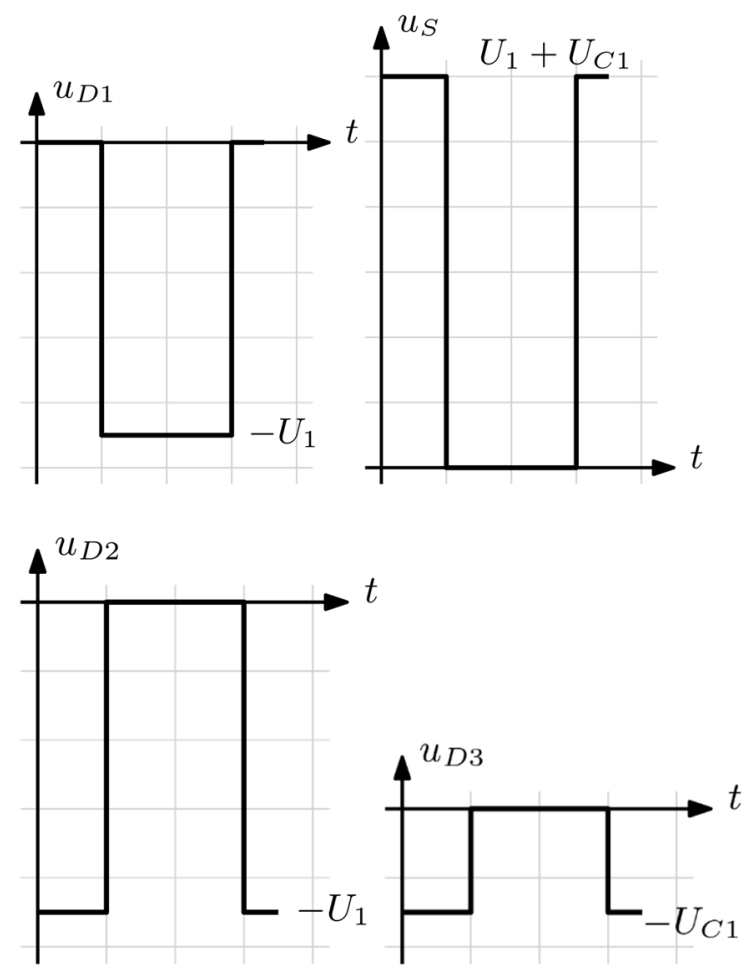

Fig. 3. Voltages across the semiconductors

Starting from the output current (Fig. 4) and using the charge balance across the capacitors (the current through the capacitors must be zero in the steady state mode), the connections between the currents can be found.

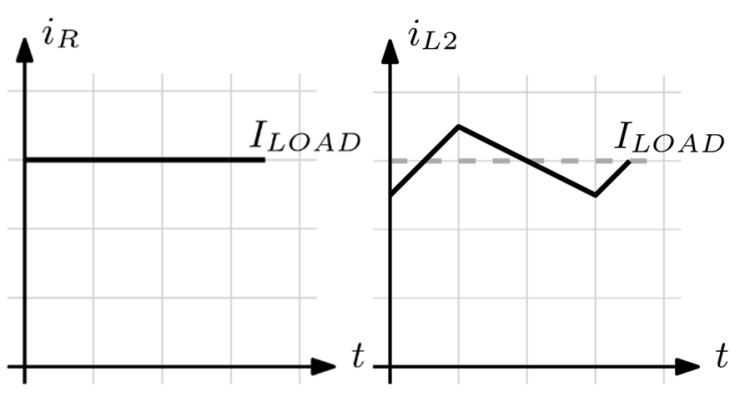

Fig. 4. Currents through the load and through the inductor $\mathrm{L}_{2}$
Starting from the fact that all charge transported through $\mathrm{L}_{2}$ must be transferred into the load, the mean value of the inductor current must be equal to the load current. The ripple of the inductor current must be equalized by capacitor $\mathrm{C}_{2}$.

With the charge balance of $\mathrm{C}_{1}$, one can also find the mean value of the current through $L_{1}$. The current through $\mathrm{L}_{1}$ increases, when the active switch is on and therefore a positive voltage is across $L_{1}$ and decreases, when the active switch is turned off and a negative voltage is across $L_{1}$. The drawings are shown in Fig. 5.

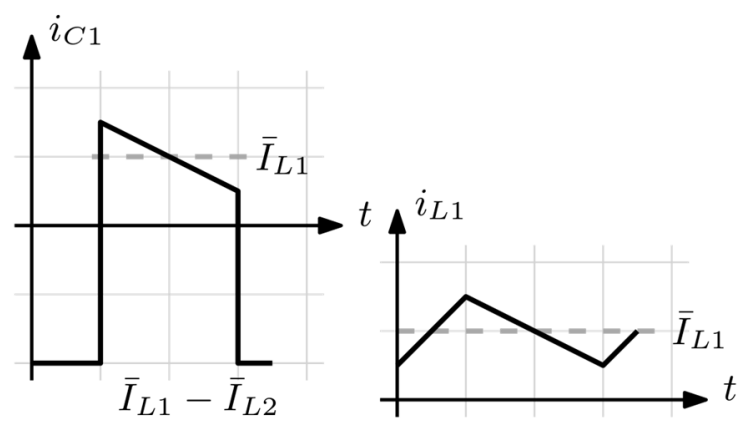

Fig. 5. Currents through $\mathrm{C}_{1}$ and $\mathrm{L}_{1}$

The current through the active switch is the current through $\mathrm{L}_{2}$ when the switch is on and zero when it is off, the current through $\mathrm{D}_{1}$ is the difference between the current through $\mathrm{L}_{2}$ and $\mathrm{L}_{1}$, when the diode is conducting during $\mathrm{M}_{1} . \mathrm{D}_{2}$ has to conduct the current through $L_{1}$ and $D_{3}$ has to conduct the current through $L_{2}$, when the active switch is off. The sketches are shown in Fig. 6. The constant current through $\mathrm{D}_{1}$ is caused by the fact that the value of the current ripples through the inductors are chosen the same. This means that in these diagrams the value of $L_{2}$ is a third of that of $L_{1}$.
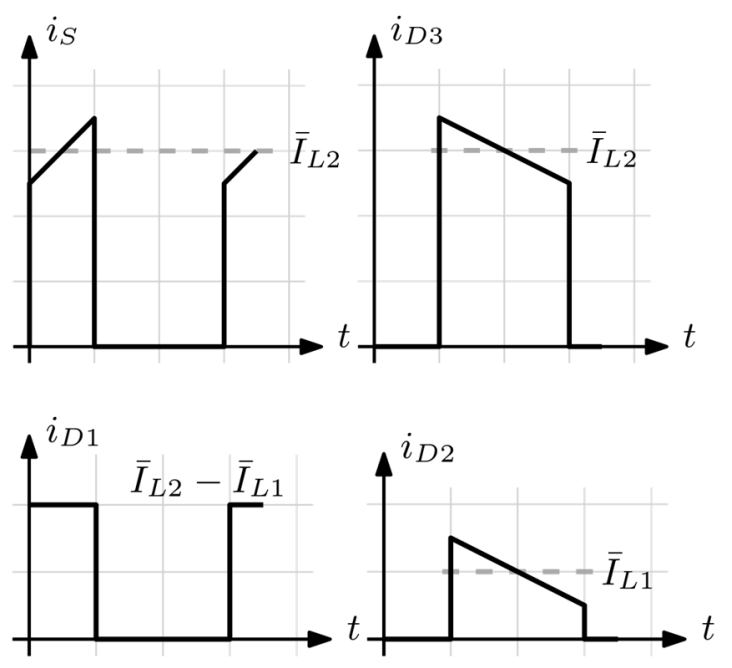

Fig. 6. Currents through the semiconductors 
For the connection of the mean values of the inductor currents one gets from Fig. 5 and the charge balance

$$
\begin{aligned}
& \left|\bar{I}_{L 1}-\bar{I}_{L 2}\right| d=\bar{I}_{L 1}(1-d) \\
& \bar{I}_{L 1}=d \cdot \bar{I}_{L 2}=d \cdot I_{L O A D}
\end{aligned}
$$

\section{Model of the D-square step-down converter}

\subsection{State equations}

The active switch $\mathrm{S}$ and the passive switch $\mathrm{D}_{1}$ are conducting during mode M1 (Fig. 7).

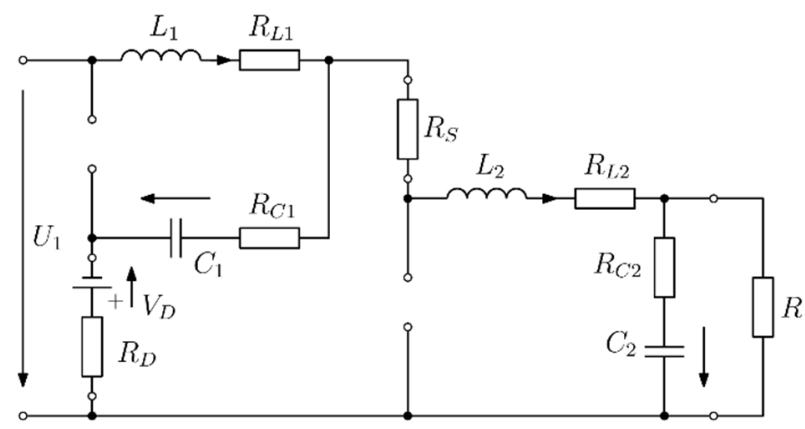

Fig. 7. Equivalent circuit of mode M1

Kirchhoff's voltage law (KVL) and sorting according to the state and input variables leads for the derivation of the current through $\mathrm{L}_{1}$ according to

$\frac{d i_{L 1}}{d t}=\frac{-i_{L 1}\left(R_{C 1}+R_{D}+R_{L 1}\right)+\left(R_{C 1}+R_{D}\right) i_{L 2}-u_{C 1}+u_{1}+V_{D}}{L_{1}}$

KVL results for the derivative of the current through the output inductor to

$$
\frac{d i_{L 2}}{d t}=\frac{1}{L_{2}}\left[\begin{array}{l}
-R_{S} i_{L 2}+\left(i_{L 1}-i_{L 2}\right)\left(R_{C 1}+R_{D}\right)+ \\
u_{C 1}-V_{D}-u_{C 2}-R_{C 2} i_{C 2}-R_{L 2} i_{L 2}
\end{array}\right] .
$$

The current through $\mathrm{C}_{2}$ is neither a state nor an input variable. Therefore, it must be substituted by

$$
i_{C 2}=\frac{R i_{L 2}-u_{C 2}}{R+R_{C 2}}
$$

This leads to

$$
\frac{d i_{L 2}}{d t}=\frac{1}{L_{2}}\left[\begin{array}{l}
\left(R_{C 1}+R_{D}\right) i_{L 1}-\left(\begin{array}{l}
R_{C 1}+R_{D}+R_{L 2} \\
+R_{S}+R / / R_{C 2}
\end{array}\right) i_{L 2} \\
+u_{C 1}-\frac{R}{R+R_{C 2}} u_{C 2}-V_{D}
\end{array}\right]
$$

For the derivatives of the voltages across the capacitors one can write

$$
\begin{aligned}
& \frac{d u_{C 1}}{d t}=\frac{i_{L 1}-i_{L 2}}{C_{1}} \\
& \frac{d u_{C 2}}{d t}=\frac{R i_{L 2}-u_{C 2}}{C_{2}\left(R+R_{C 2}\right)} .
\end{aligned}
$$

For mode M2 (Fig. 8) one gets

$$
\begin{gathered}
\frac{d i_{L 1}}{d t}=\frac{-i_{L 1}\left(R_{C 1}+R_{D}+R_{L 1}\right)-u_{C 1}-V_{D}}{L_{1}} \\
\frac{d i_{L 2}}{d t}=\frac{1}{L_{2}}\left[\begin{array}{l}
-\left(R_{D}+R_{L 2}+R / / R_{C 2}\right) i_{L 2}-\frac{R}{R+R_{C 2}} u_{C 2} \\
-V_{D} \\
\frac{d u_{C 1}}{d t}=\frac{i_{L 1}}{C_{1}}
\end{array}\right.
\end{gathered}
$$

The differential equation for $\mathrm{C}_{2}$ is the same one as in mode M1.

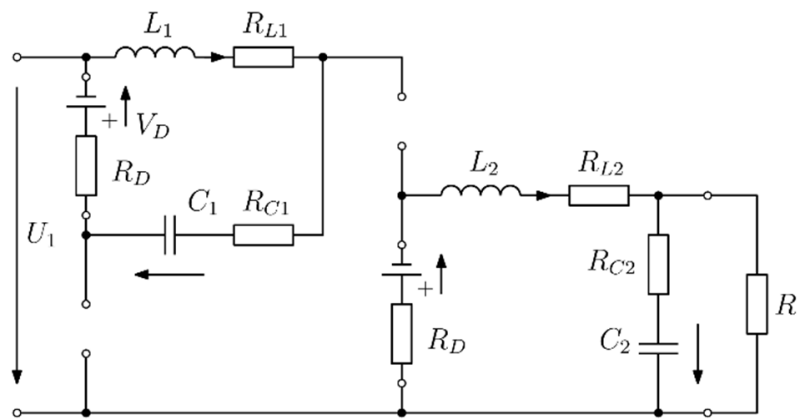

Fig. 8 Equivalent circuit of mode M2

\subsection{Large signal model}

Combining these equations (state space averaging: the equations for mode M1 are weighted by the duty cycle $\mathrm{d}$, the equations for mode $\mathrm{M} 2$ are multiplied by $1-\mathrm{d}$ and added) leads to the large signal model according to the result shown in appendix A1.

\subsection{Small signal model}

Using the perturbation concept (each variable in the large signal model is written as the value at the working point, marked as capital letters and a zero in the index, added by a small disturbance, written with small letters with a roof on top. To get a linearized equation the products of disturbances are omitted) one gets the small signal model around the working point described by $\mathrm{U}_{10}, \mathrm{I}_{\mathrm{L} 10}, \mathrm{I}_{\mathrm{L} 20}, \mathrm{U}_{\mathrm{C} 10}, \mathrm{U}_{\mathrm{C} 20}$ according to the equation given in appendix A2.

When simplified with abbreviations the model can be written as (15)

$$
\frac{d}{d t}\left(\begin{array}{l}
\hat{i}_{L 1} \\
\hat{i}_{L 2} \\
\hat{u}_{C 1} \\
\hat{u}_{C 2}
\end{array}\right)=\left[\begin{array}{cccc}
A_{11} & A_{12} & A_{13} & 0 \\
A_{21} & A_{22} & A_{23} & A_{24} \\
A_{31} & A_{32} & 0 & 0 \\
0 & A_{42} & 0 & A_{44}
\end{array}\right]\left(\begin{array}{l}
\hat{i}_{L 1} \\
\hat{i}_{L 2} \\
\hat{u}_{C 1} \\
\hat{u}_{C 2}
\end{array}\right)+\left[\begin{array}{cc}
B_{11} & B_{12} \\
0 & B_{22} \\
0 & B_{32} \\
0 & 0
\end{array}\right]\left(\begin{array}{c}
\hat{u} \\
\hat{d}
\end{array}\right)
$$

Laplace transformation leads to (16) 


$$
\left[\begin{array}{cccc}
s-A_{11} & -A_{12} & -A_{13} & 0 \\
-A_{21} & s-A_{22} & -A_{23} & -A_{24} \\
-A_{31} & -A_{32} & s & 0 \\
0 & -A_{42} & 0 & s-A_{44}
\end{array}\right]\left(\begin{array}{c}
I_{L 1}(s) \\
I_{L 2}(s) \\
U_{C 1}(s) \\
U_{C 2}(s)
\end{array}\right)=\left[\begin{array}{cc}
B_{11} & B_{12} \\
0 & B_{22} \\
0 & B_{32} \\
0 & 0
\end{array}\right]\left(\begin{array}{c}
U_{1}(s) \\
D(s)
\end{array}\right)
$$

\subsection{Transfer functions}

From (16) one can calculate eight transfer functions. For a DC/DC converter the output voltage in dependence of the duty cycle (for the control) and in dependence on the input voltage (for the disturbance) are the most important ones. In our case (when the parasitic resistances are included in the model) also the transfer functions for the current through $\mathrm{L}_{2}$ are necessary. The easiest way to achieve the numerators and the denominator is to use Crammer's law. The denominator is the same for all cases and results in the equation given in appendix A3.

The numerators for

$$
\begin{aligned}
& \frac{U_{C 2}(s)}{D(s)}=\frac{N_{-} U_{C 2} D}{D(s)}, \frac{U_{C 2}(s)}{U_{1}(s)}=\frac{N_{-} U_{C 2} U_{1}}{U_{1}(s)}, \\
& \frac{I_{L 2}(s)}{D(s)}=\frac{N_{-} U_{L 2} D}{D(s)}, \frac{I_{L 2}(s)}{U_{1}(s)}=\frac{N_{-} I_{L 2} U_{1}}{U_{1}(s)}
\end{aligned}
$$

are (18)

$$
\begin{aligned}
& N_{-} U_{C 2} D=s^{2} A_{42} B_{22}+s A_{42}\left(\begin{array}{l}
A_{23} B_{32}+A_{21} B_{12} \\
-A_{11} B_{22}
\end{array}\right) \\
& -A_{42}\left(\begin{array}{l}
A_{11} A_{23} B_{32}+A_{13} A_{31} B_{22} \\
-A_{13} A_{21} B_{32}-A_{23} A_{31} B_{12}
\end{array}\right) \\
& N_{-} U_{C 2} U_{1}=B_{11} A_{42}\left(s A_{21}+A_{23} A_{31}\right) \\
& N_{-} I_{L 2} D=s^{3} B_{22}+s^{2}\left(\begin{array}{l}
-A_{11} B_{22}+A_{21} B_{12}+A_{23} B_{32} \\
-A_{44} B_{22}
\end{array}\right)+ \\
& +s\left(\begin{array}{l}
A_{23} A_{31} B_{12}+A_{13} A_{21} B_{32}-A_{13} A_{31} B_{22} \\
-A_{11} A_{23} B_{12}+ \\
A_{44}\left(A_{11} B_{22}-A_{21} B_{12}-A_{23} B_{12}\right)
\end{array}\right)- \\
& -A_{44}\left(\begin{array}{l}
A_{23} A_{31} B_{12}+A_{13} A_{21} B_{32}-A_{13} A_{31} B_{22} \\
-A_{11} A_{23} B_{32}
\end{array}\right) \\
& N_{-} I_{L 2} U_{1}=s^{2} A_{21} B_{11}+s\left(A_{23} A_{31} B_{11}-A_{21} A_{44} B_{11}\right), \\
& +A_{23} A_{31} A_{44} B_{11}
\end{aligned}
$$

respectively.

\subsection{Stationary case}

Starting from the large-signal model (A1) and substituting for all variables the value of the working point $F_{0}$ and a small disturbance $\hat{f}$ in the form $f=F_{0}+\hat{f}$, one can calculate the connections between the working point values $\mathrm{I}_{\mathrm{L} 10}, \mathrm{I}_{\mathrm{L} 20}, \mathrm{U}_{\mathrm{C} 10}$, $\mathrm{U}_{\mathrm{C} 20}$ in relation to the input values $\mathrm{U}_{10}, \mathrm{D}_{0}$. The derivative of the functions on the left side of (A1) shows that for the equations for the working point values the left side is zero and on the right side only elements marked by a zero have to be taken into account

$$
\begin{gathered}
0=-\frac{R_{C 1}+R_{D}+R_{L 1}}{L_{1}} I_{L 10}+\frac{\left(R_{C 1}+R_{D}\right)}{L_{1}} D_{0} I_{L 20} \\
-\frac{1}{L_{1}} U_{C 10}+\frac{1}{L_{1}} D_{0} U_{10}+\frac{V_{D}}{L_{1}} \\
0=\frac{\left(R_{C 1}+R_{D}\right)}{L_{2}} D_{0} I_{L 10}-\frac{\left(R_{L 1}+R / / R_{C 2}+D_{0}\right.}{L_{2}} I_{L 20}+ \\
\frac{1}{L_{2}} D_{0} U_{C 10}-\frac{R}{L_{2}\left(R+R_{C 2}\right)} U_{C 20}-\frac{V_{D}}{L_{2}}
\end{gathered}
$$

$$
\begin{aligned}
& 0=\frac{1}{C_{1}} I_{L 10}-\frac{1}{C_{1}} D_{0} I_{L 20} \\
& 0=\frac{R}{C_{2}\left(R+R_{C 2}\right)} I_{L 20}-\frac{1}{C_{2}\left(R+R_{C 2}\right)} U_{C 20} .
\end{aligned}
$$

These four linear equations result in

$$
\begin{aligned}
& I_{L 10}=D_{0} I_{L 20}=\frac{D_{0} U_{C 20}}{R}=D_{0} I_{\text {Load }} \\
& I_{L 20}=\frac{U_{C 20}}{R}=I_{\text {Load }} .
\end{aligned}
$$

The equations for the voltages across the capacitors are given because of their largeness in appendix 4 . Calculation of the output voltage leads to

$$
U_{20}=R / / R_{C 2} \cdot I_{L 20}+\frac{R}{R+R_{C 2}} \cdot U_{C 20} .
$$

Fig. 9 shows a Bode plot and Fig. 10 shows the step response to a duty cycle step (the parameters are $\mathrm{L}_{1}=\mathrm{L}_{2}=47 \mu \mathrm{H}, \quad \mathrm{C}_{1}=\mathrm{C}_{2}=330 \mu \mathrm{F}, \quad \mathrm{R}_{\mathrm{L} 1}=\mathrm{R}_{\mathrm{L} 2}=4 \mathrm{~m} \Omega$, $\mathrm{R}_{\mathrm{Cl}}=\mathrm{R}_{\mathrm{C} 2}=80 \mathrm{~m} \Omega, \mathrm{R}_{\mathrm{S}}=10 \mathrm{~m} \Omega, \mathrm{R}_{\mathrm{D}}=0,1 \Omega, \mathrm{V}_{\mathrm{D}}=1 \mathrm{~V}$ $\mathrm{U}_{10}=24 \mathrm{~V}, \mathrm{R}=0,5 \Omega$ ). The converter is a phaseminimum system and has two zeros (19) and four poles (A3).

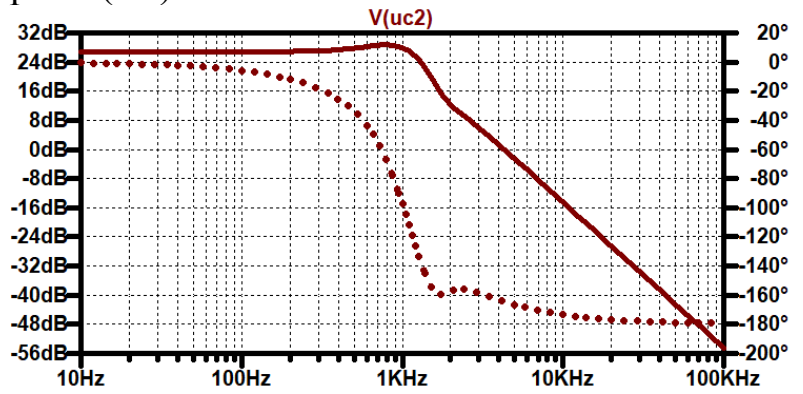

Fig 9. Bode plot voltage across $\mathrm{C}_{2}$ in dependence of the duty cycle 
Fig. 10 depicts the step response. On the left diagram, calculated with the transfer function, one can see only the change of the output voltage referred to starting working point. To get the real value one has to add the initial value. The result on the right side, achieved by circuit simulation, shows the actual value. The ripple is caused by the series resistor of the output capacitor $\mathrm{C}_{2}$.

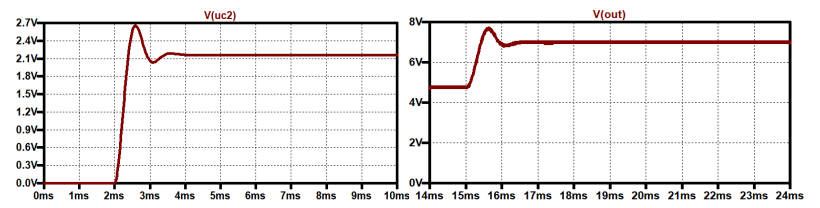

Fig. 10. Duty cycle step response, left: linearized around the working point, right: circuit oriented simulation.

\section{Feedforward control}

\subsection{Control law}

From the idealized voltage transformation ratio (3) one can, with $U_{\text {ref }}$ as the command, obtain the control law

$$
d=\sqrt{\frac{U_{2 r e f}}{U_{1}}} .
$$

\subsection{Linearized control law}

To obtain a linearized control law, we use again the perturbation concept leading to

$$
\begin{gathered}
D_{0}+\hat{d}=\sqrt{\frac{U_{2 r e f 0}+\hat{u}_{2 r e f}}{U_{10}+\hat{u}_{1}}} \\
D_{0}+\hat{d}=\sqrt{\frac{U_{2 \text { ref } 0}}{U_{101}}} \sqrt{\frac{1+\frac{\hat{u_{2 r e f}}}{U_{2 r e f 0}}}{1+\frac{\hat{u_{1}}}{U_{10}}}}
\end{gathered}
$$

With the approximations for an $\varepsilon$ much smaller than one

$$
\begin{aligned}
& \sqrt{1 \pm \varepsilon}=1 \pm \frac{\varepsilon}{2} \\
& \frac{1}{\sqrt{1 \pm \varepsilon}}=1 \mp \frac{\varepsilon}{2}
\end{aligned}
$$

one can write

$$
D_{0}+\hat{d}=\sqrt{\frac{U_{2 \text { ref } 0}}{U_{10}}}\left(1+\frac{\hat{u}_{2 \text { ref }}}{2 U_{2 \text { ref } 0}}\right)\left(1-\frac{\hat{u_{1}}}{2 U_{10}}\right)
$$

Multiplying and omitting the quadratic term, one gets for the linearized duty cycle (35)
$D_{0}+\hat{d}=\sqrt{\frac{U_{2 \text { refo } 0}}{U_{10}}}+\frac{1}{2 \sqrt{U_{10} U_{2 \text { ref } 0}}} \hat{u}_{2 \text { ref }}-\frac{1}{2 U_{10}} \sqrt{\frac{U_{2 \text { ref } 0}}{U_{10}}} \hat{u}_{1}$

Now we can include, with abbreviations for the disturbance terms $G_{1}$ and $G_{2}$, the perturbation of the duty cycle

$$
\hat{d}=G_{1} \hat{u}_{2 \text { ref }}+G_{2} \hat{u}_{1}
$$

into (15) and obtain the small signal model of the feed-forward controlled d-square converter

$$
\begin{aligned}
\frac{d}{d t}\left(\begin{array}{l}
\hat{i}_{L 1} \\
\hat{i}_{L 2} \\
\hat{u}_{C 1} \\
\hat{u}_{C 2}
\end{array}\right) & {\left[\begin{array}{cccc}
A_{11} & A_{12} & A_{13} & 0 \\
A_{21} & A_{22} & A_{23} & A_{24} \\
A_{31} & A_{32} & 0 & 0 \\
0 & A_{42} & 0 & A_{44}
\end{array}\right]\left(\begin{array}{l}
\hat{i}_{L 1} \\
\hat{i_{L 2}} \\
\hat{u}_{C 1} \\
\hat{u}_{C 2}
\end{array}\right)+} \\
& {\left.\left[\begin{array}{cc}
B_{11} & B_{12} \\
0 & B_{22} \\
0 & B_{32} \\
0 & 0
\end{array}\right] \begin{array}{c}
\hat{u}_{1} \\
G_{1} \hat{u}_{2 r e f}+G_{2} \hat{u}_{1}
\end{array}\right) }
\end{aligned}
$$

This leads to the small signal model of the feedforward controlled d-square converter according to

$$
\begin{aligned}
\frac{d}{d t}\left(\begin{array}{l}
\hat{i}_{L 1} \\
\hat{i}_{L 2} \\
\hat{u}_{C 1} \\
\hat{u}_{C 2}
\end{array}\right) & =\left[\begin{array}{cccc}
A_{11} & A_{12} & A_{13} & 0 \\
A_{21} & A_{22} & A_{23} & A_{24} \\
A_{31} & A_{32} & 0 & 0 \\
0 & A_{42} & 0 & A_{44}
\end{array}\right]\left(\begin{array}{l}
\hat{i}_{L 1} \\
\hat{i}_{L 2} \\
\hat{u}_{C 1} \\
\hat{u}_{C 2}
\end{array}\right) \\
& +\left[\begin{array}{cc}
B_{11}+B_{12} G_{2} & B_{12} G_{1} \\
B_{22} G_{2} & B_{22} G_{1} \\
B_{32} G_{2} & B_{32} G_{1} \\
0 & 0
\end{array}\right]\left(\begin{array}{c}
\hat{u}_{1} \\
\hat{u}_{2 \text { ref }}
\end{array}\right)
\end{aligned}
$$

(38) describes the system to be controlled by an additional feed-back controller. This controller has only to compensate the error of the not exact control law. Fig. 11 shows this concept. The abbreviations are: CL control law, FBC feedback controller, DC/DC represents the converter which has to be controlled.

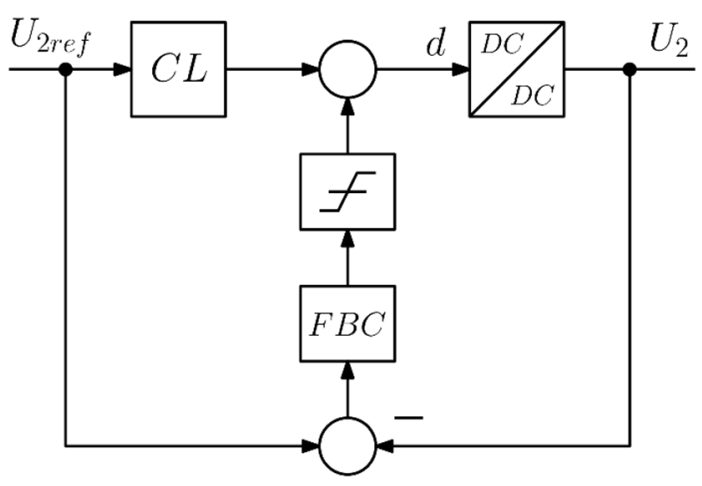

Fig. 11. Combined feedforward and feedback control 
Fig. 12 shows the start-up and the reaction to an input voltage of the converter step after $15 \mathrm{~ms}$ using the simple control law. At the beginning the converter is in discontinuous mode. This is visible by the fact that the output voltage is higher than the reference value. The steady-state error is caused by the parasitic resistances of the converter.

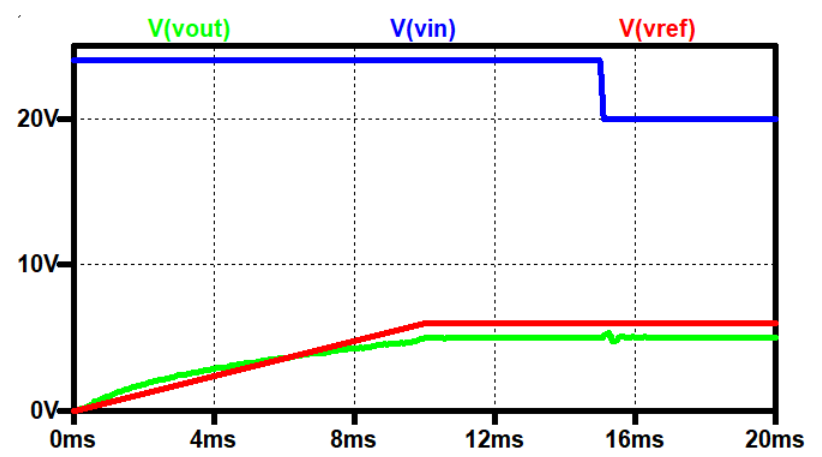

Fig. 12. input voltage (blue), reference value (red), output voltage (green)

The input voltage step is immediately compensated by the control low, only a small ringing occurs.

\subsection{More precise control law}

For a more precise control law with included parasitics we start from

$$
U_{20}=R / / R_{C 2} \cdot I_{L 20}+\frac{R}{R+R_{C 2}} \cdot U_{C 20}
$$

and including (27) and (A4.2) one can write

$$
\begin{aligned}
& U_{C 2 \text { ref }}=\frac{-D_{0}^{2} U_{10}-V_{D} D_{0}+V_{D}}{a_{2} \cdot D_{0}^{2}+a_{1} \cdot D_{0}+a_{0}} \\
& a_{2}=\frac{\left(R_{C 1}+R_{D}\right)-\left(R_{L 1}+R_{C 1}+R_{D}\right)}{R}=-\frac{R_{L 1}}{R} \\
& a_{1}=\frac{-\left(R_{C 1}+R_{S}\right)+\left(R_{C 1}+R_{D}\right)}{R}=\frac{R_{D}-R_{S}}{R} \\
& a_{0}=-\left(\frac{R_{D}+R_{L 2}+R / / R_{C 2}}{R}+\frac{R}{\left(R+R_{C 2}\right)}\right)
\end{aligned}
$$

Rearranging leads to the control law

$$
d=-\frac{a_{1} U_{C 2 \text { ref }}+V_{D}}{2\left(U_{C 2 \text { ref }} a_{2}+U_{1}\right)}+\sqrt{\begin{array}{l}
\left(\frac{a_{1} U_{C 2 r e f}+V_{D}}{2\left(U_{C 2 r e f} a_{2}+U_{1}\right)}\right)^{2} \\
-\frac{a_{0} U_{C 2 r e f}-V_{D}}{U_{C 2 r e f} a_{2}+U_{1}}
\end{array}}
$$

This concept has to be implemented in a digital way. Fig. 13 shows the results for the precise control law. The improvement can be seen easily. Now only changes in the parasitic resistors (influence of the temperature and tolerances) lead to an imprecise output voltage.

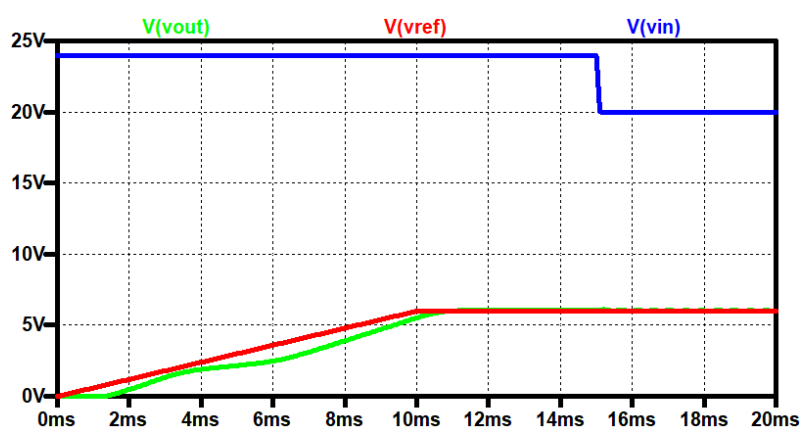

Fig. 13 input voltage (blue), reference value (red), output voltage (green)

\section{Examples of other Quadratic Converters}

In this chapter some other quadratic converters are shown to prove the efficiency of the simple feedforward control.

\subsection{Quadratic step-down converter II}

Fig. 14 shows another possibility to realize a quadratic step-down converter.

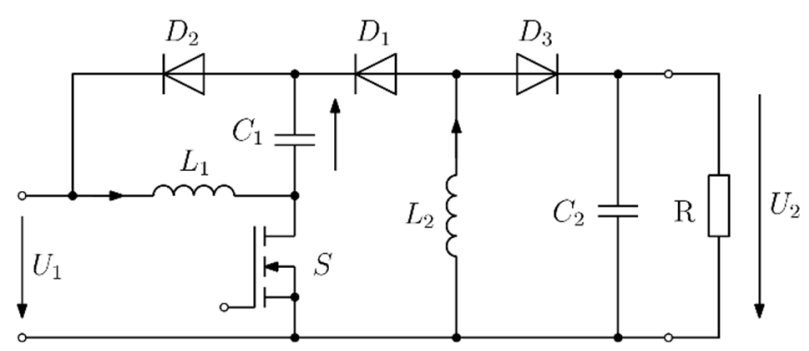

Fig. 14. Quadratic step-down converter II

From the voltage transformation ratio

$$
U_{2}=\left(\frac{d}{1-d}\right)^{2} U_{1}
$$

one gets the quadratic equation

$$
d^{2}+\frac{2 U_{2}}{U_{1}-U_{2}} d-\frac{U_{2}}{U_{1}-U_{2}}=0 .
$$

Solving this equation leads to the control law

$$
d=\frac{U_{2}}{U_{1}-U_{2}}\left(-1+\sqrt{\frac{U_{1}}{U_{2}}}\right) .
$$

It should be mentioned, that the output voltage must be lower than the input voltage, therefore the converter is only a step-down converter with a quadratic step-up-down transformation rate! This effect can also be found, when one calculates the voltage across $\mathrm{D}_{1}$, but cannot be seen, when one considers the voltage transformation ratio alone! The maximum duty cycle can be calculated according to $d_{\max }=0.5$. 
Fig. 15 shows the start-up and the reaction of the feedforward controlled converter to an input voltage step.

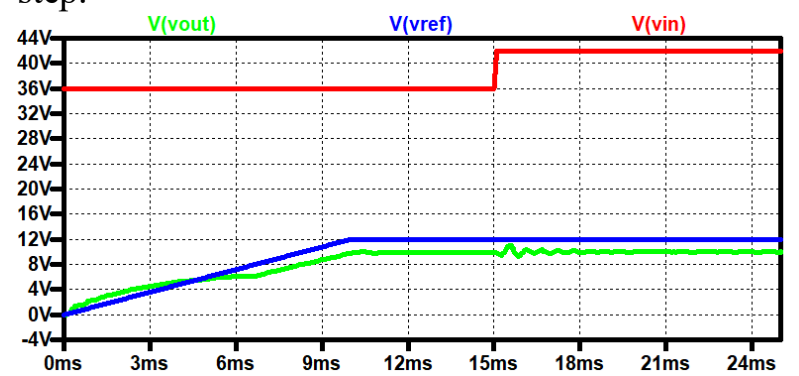

Fig. 15. Input voltage (red), reference value (blue), output voltage (green)

\subsection{Inverting quadratic step-up-down converter}

Fig. 16 shows the circuit diagram of an inverting quadratic step-up-down converter.

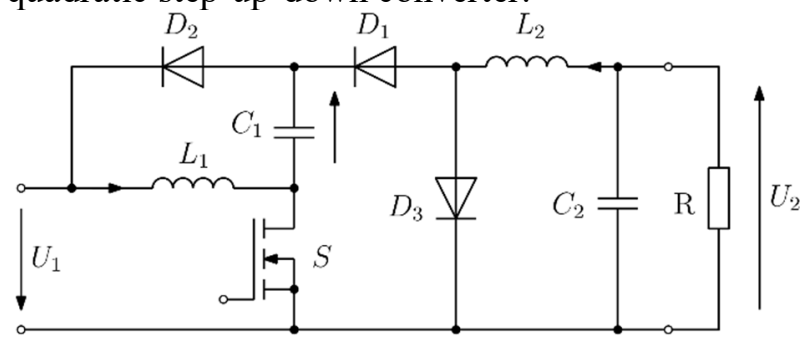

Fig. 16. Inverting quadratic step-up-down converter

The voltage transformation ratio of this converter

$$
U_{2}=\frac{d^{2}}{1-d} U_{1}
$$

leads to a quadratic equation in $\mathrm{d}$

$$
d^{2}+d \frac{U_{2}}{U_{1}}-\frac{U_{2}}{U_{1}}=0
$$

This leads to the idealized control law

$$
d=\frac{U_{2 r e f}}{2 U_{1}}\left(-1+\sqrt{1+\frac{4 U_{1}}{U_{2 r e f}}}\right)
$$

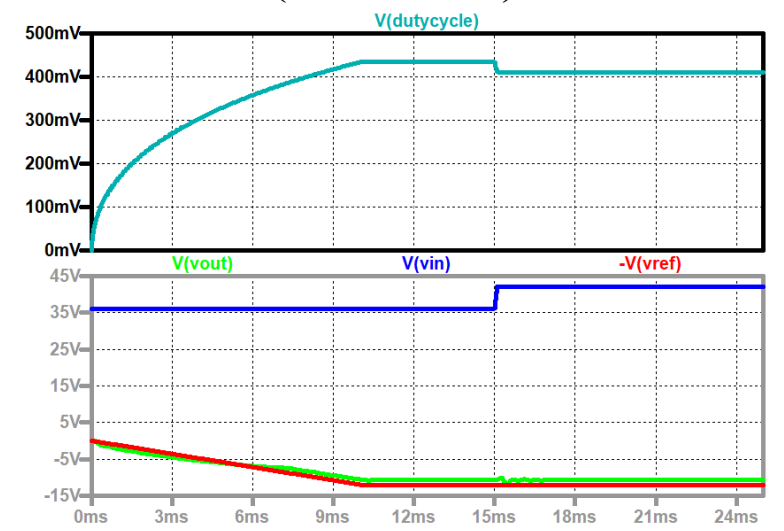

Fig. 17. duty cycle (turquoise); input voltage (blue); output voltage (green), reference voltage (red)

Fig. 17 shows the signal which generates the duty cycle, and the input voltage, the reference value and the obtained output voltage. The control law leads to a stable output voltage with small error.

\section{Conclusion}

Quadratic converters are interesting alternative topologies, when wide transformation ratios have to be achieved. A feedforward control with a control law, which is derived from the voltage transformation ratio, leads to a stable design and to an output voltage with only small error. To avoid this error one can calculate a more precise control law by including the parasitic resistors of the devices. This will lead still to a (small) error, because of the tolerances and the temperature dependence on the parasitics. Another way to get a correct output voltage is to include the control law into the model of the converter and design a feedback controller for this system. This feedback controller contributes only with about $5 \%$ of the necessary duty cycle.

References:

[1] D. Maksimovic and S. Cuk, Switching converters with wide DC conversion range, IEEE Transactions on Power Electronics, Vol. 6, No. 1, pp. 151-157, Jan. 1991.

[2] N. Mohan, T. Undeland and W. Robbins, Power Electronics, Converters, Applications and Design, 3nd ed. New York: W. P. John Wiley \& Sons, 2003.

[3] F. Zach, Power Electronics, in German: Leistungselektronik, Wien: Springer, 6th ed., 2021.

[4] Y. Rozanov, S. Ryvkin, E. Chaplygin, P. Voronin, Power Electronics Basics, CRC Press, 2016.

[5] S. Cuk, General topological properties of switching structures, IEEE Power Electronics Specialists Conference, San Diego, CA, USA, 1979, pp. 109-130.

[6] B. W. Williams, Generation and Analysis of Canonical Switching Cell DC-to-DC Converters, IEEE Transactions on Industrial Electronics, Vol. 61, No. 1, pp. 329-346, Jan. 2014.

[7] R. Marquez and M. A. Contreras-Ordaz, The Three-Terminal Converter Cell, Graphs, and Generation of DC-to-DC Converter Families, IEEE Transactions on Power Electronics, Vol. 35, No. 8, pp. 7725-7728, Aug. 2020

[8] F. A. Himmelstoss and H. L. Votzi, A family. of quadratic DC/DC converters with one low-side switch and a tapped inductor at the output side, 2019 International Aegean Conference on Electrical Machines and Power Electronics $(A C E M P) \& 2019$ International Conference on Optimization of Electrical and Electronic Equipment (OPTIM), Istanbul, Turkey, 2019, pp. 304-309

[9] F. A. Himmelstoss and K. H. Edelmoser, Quadratic Flyback Converter, PCIM Europe digital days 2020; International Exhibition and Conference for Power Electronics, Intelligent 
Motion, Renewable Energy and Energy Management, 2020, pp. 1-6.

[10] F. A. Himmelstoss, H. L. Votzi and M. Windisch, Quadratic DC/DC converter with autotransformer at the output side, 2020 IEEE 29th International Symposium on Industrial Electronics (ISIE), 2020, pp. 767-772.
[11] F. A. Himmelstoss and H. L. Votzi, side switch and a tapped inductor at the input side, IECON 2019 - 45th Annual Conference of the IEEE Industrial Electronics Society, 2019, pp. 1774-1779.

\section{Appendix}

Appendix 1: Large signal model of the quadratic Buck converter

$$
\frac{d}{d t}\left(\begin{array}{c}
i_{L 1} \\
i_{L 2} \\
u_{C 1} \\
u_{C 2}
\end{array}\right)=\left[\begin{array}{cccc}
-\frac{R_{C 1}+R_{D}+R_{L 1}}{L_{1}} & \frac{\left(R_{C 1}+R_{D}\right) d}{L_{1}} & -\frac{1}{L_{1}} & 0 \\
\frac{\left(R_{C 1}+R_{D}\right) d}{L_{2}} & -\frac{R_{D}+R_{L 2}+R / / R_{C 2}+\left(R_{C 1}+R_{S}\right) d}{L_{2}} & \frac{d}{L_{2}} & -\frac{R}{L_{2}\left(R+R_{C 2}\right)} \\
\frac{1}{C_{1}} & -\frac{d}{C_{1}} & 0 & 0 \\
0 & \frac{R}{C_{2}\left(R+R_{C 2}\right)} & 0 & -\frac{1}{C_{2}\left(R+R_{C 2}\right)}
\end{array}\right]\left(\begin{array}{c}
i_{L 1} \\
i_{L 2} \\
u_{C 1} \\
u_{C 2}
\end{array}\right)+\left[\begin{array}{c}
\frac{d}{L_{1}} \\
0 \\
0 \\
0
\end{array}\right]\left(u_{1}\right)+\left(\begin{array}{c}
\frac{V_{D}}{L_{1}} \\
-\frac{V_{D}}{L_{2}} \\
0 \\
0
\end{array}\right)
$$

Appendix 2: Small signal model of the quadratic Buck converter

$$
\begin{aligned}
& \frac{d}{d t}\left(\begin{array}{l}
\hat{i}_{L 1} \\
\hat{i}_{L 2} \\
\hat{u}_{C 1} \\
\hat{u}_{C 2}
\end{array}\right) {\left[\begin{array}{cccc}
-\frac{R_{C 1}+R_{D}+R_{L 1}}{L_{1}} & \frac{\left(R_{C 1}+R_{D}\right) D_{0}}{L_{1}} & -\frac{1}{L_{1}} & 0 \\
\frac{\left(R_{C 1}+R_{D}\right) D_{0}}{L_{2}} & -\frac{R_{D}+R_{L 2}+R / / R_{C 2}+\left(R_{C 1}+R_{S}\right) D_{0}}{L_{2}} & \frac{D_{0}}{L_{2}} & -\frac{R}{L_{2}\left(R+R_{C 2}\right)} \\
\frac{1}{C_{1}} & -\frac{D_{0}}{C_{1}} & 0 & 0 \\
0 & \frac{R}{C_{2}\left(R+R_{C 2}\right)} \\
+ & 0 & -\frac{1}{C_{2}\left(R+R_{C 2}\right)}
\end{array}\right]\left(\begin{array}{l}
\hat{i}_{L 1} \\
\hat{i}_{L 2} \\
\hat{u}_{C 1} \\
\hat{u}_{C 2}
\end{array}\right) } \\
&+\left[\begin{array}{cc}
\frac{D_{0}}{L_{1}} & \frac{\left(R_{C 1}+R_{D}\right) I_{L 20}+U_{10}}{L_{1}} \\
0 & \frac{\left(R_{C 1}+R_{D}\right) I_{L 10}-\left(R_{C 1}+R_{S}\right) I_{L 20}+U_{C 10}}{L_{2}} \\
0 & -\frac{I_{L 20}}{C_{1}} \\
0 & 0
\end{array}\right]\left(\begin{array}{l}
\hat{u_{1}} \\
\hat{d}
\end{array}\right) .
\end{aligned}
$$

Appendix 3: Denominator of the transfer functions of the quadratic Buck converter

$D=s^{4}-s^{3}\left(A_{44}+A_{11}+A_{22}\right)+s^{2}\left(-A_{24} A_{42}-A_{13} A_{31}-A_{23} A_{32}+A_{11} A_{22}-A_{12} A_{21}+\left(A_{11} A_{44}+A_{22} A_{44}\right)\right)+$

$+s\left(A_{11} A_{24} A_{42}-A_{31}\left(A_{12} A_{23}-A_{13} A_{22}\right)+A_{13} A_{31} A_{44}+A_{23} A_{32} A_{44}+A_{32}\left(A_{11} A_{23}-A_{13} A_{21}\right)-A_{44}\left(A_{11} A_{22}-A_{12} A_{21}\right)\right)+$

$+\left(A_{13} A_{24} A_{31} A_{42}-A_{44}\left(-A_{31} A_{12} A_{23}+A_{13} A_{22} A_{31}+A_{11} A_{23} A_{32}-A_{13} A_{21} A_{32}\right)\right)$

Appendix 4: Stationary values for the voltages across the capacitors

$$
\begin{aligned}
U_{C 10}= & \frac{\left(D_{0} U_{10}+V_{D}\right)\left(\frac{\left(R_{C 1}+R_{D}\right)}{R} D_{0}^{2}-\frac{R_{D}+R_{L 2}+R / / R_{C 2}+\left(R_{C 1}+R_{S}\right) D_{0}}{R}-\frac{R}{\left(R+R_{C 2}\right)}\right)+V_{D} \frac{\left(R_{C 1}+R_{D}\right)\left(1-D_{0}\right)-R_{L 1} D_{0}}{R}}{\left[\frac{\left(R_{C 1}+R_{D}\right)}{R} D_{0}^{2}-\frac{R_{D}+R_{L 2}+R / / R_{C 2}+\left(R_{C 1}+R_{S}\right) D_{0}}{R}-\frac{R}{\left(R+R_{C 2}\right)}\right]+D_{0} \frac{\left(R_{C 1}+R_{D}\right)\left(1-D_{0}\right)-R_{L 1} D_{0}}{R}} \\
U_{C 20}= & \frac{V_{D}-\left(D_{0} U_{10}+V_{D}\right) D_{0}}{\frac{\left(R_{C 1}+R_{D}\right)}{R} D_{0}^{2}-\frac{R_{D}+R_{L 2}+R / / R_{C 2}+\left(R_{C 1}+R_{S}\right) D_{0}}{R}-\frac{R}{\left(R+R_{C 2}\right)}+D_{0} \frac{\left(R_{C 1}+R_{D}\right)\left(1-D_{0}\right)-R_{L 1} D_{0}}{R}}
\end{aligned}
$$


Contribution of Individual Authors to the Creation of a Scientific Article (Ghostwriting Policy)

The authors equally contributed in the present research, at all stages from the formulation of the problem to the final findings and solution.

Sources of Funding for Research Presented in a Scientific Article or Scientific Article Itself

No funding was received for conducting this study.

\section{Conflict of Interest}

The authors have no conflicts of interest to declare that are relevant to the content of this article.

Creative Commons Attribution License 4.0 (Attribution 4.0 International, CC BY 4.0)

This article is published under the terms of the Creative Commons Attribution License 4.0

https://creativecommons.org/licenses/by/4.0/deed.en 